\title{
Los pacientes con delgadez constitucional presentan un fenotipo diferente al de los pacientes con trastornos de la alimentación
}

\author{
Patients with constitutional thinness have a different phenotype than patients with eating disorders
}

Gabriel Esteban Villalon ${ }^{\mathrm{a}}$ (D)

\begin{abstract}
Resumen
Los pacientes con delgadez constitucional suelen presentar un peso habitual reducido pero estacionario en el tiempo, con dificultad para aumentarlo incluso luego de someterse a dietas hipercalóricas. A diferencia de otras entidades, no se suelen encontrar trastornos de la alimentación, enfermedades sistémicas, desnutrición o sobre-ejercitación. Sin embargo, su presencia suele despertar preocupación y motivar la búsqueda de causas patológicas asociadas, tanto por parte de los pacientes como de los profesionales de la salud.

Partiendo de la viñeta clínica de una paciente que presenta estas características, el autor realiza una búsqueda bibliográfica para intentar esclarecer cuán diferentes resultan los pacientes con delgadez constitucional de aquellos que presentan el principal diagnóstico diferencial a tener en cuenta, la anorexia nerviosa.
\end{abstract}

\begin{abstract}
Patients with constitutional thinness tend to have a reduced but stationary habitual weight over time, with difficulty to increase it even after undergoing hypercaloric diets. Unlike other entities, eating disorders, systemic diseases, malnutrition or over-exercising are not usually found. However, its presence tends to arouse concern and motivates the search for associated pathological causes, both by patients and health professionals. Based on the clinical vignette of a patient who presents these characteristics, the author performs a literature search in order to clarify how different patients with constitutional thinness are from those who present the main differential diagnosis to consider: anorexia nervosa.
\end{abstract}

Palabras clave: Delgadez, Anorexia Nerviosa, Trastornos de Alimentación y de la Ingestión de Alimentos. Keywords: Thinness, Anorexia Nervosa, Feeding and Eating Disorders.

Villalon GE. Los pacientes con delgadez constitucional presentan un fenotipo diferente al de los pacientes con trastornos de la alimentación. Evid Actual Pract Ambul. 2021;24(4):e006986. Available from: https://dx.doi.org/10.51987/EVIDENCIA.V25I1.6986.

\section{Escenario clínico}

Concurre a la consulta con su médico de cabecera una paciente de 28 años preocupada por su peso actual. Mide 1,48 m de altura y su peso histórico ronda los $40 \mathrm{~kg}$, datos que permiten estimar un índice de masa corporal (IMC) de 18,3. Recientemente fue sometida a una apendicectomía, luego de la cual su peso bajó a $34,5 \mathrm{~kg}$ (IMC 15,5) y le está costando recuperar su peso habitual. Refiere apetito conservado y alimentación variada. Sus menstruaciones son regulares. Tiene dos hijos. Sus embarazos cursaron de forma normal y durante éstos llegó a pesar alrededor de $50 \mathrm{~kg}$. No realiza actividad física ni utiliza laxantes o purgantes. Desearía pesar más. Refiere haber sido estudiada por otros colegas en el pasado, quienes descartaron la coexistencia de celiaquía mediante detección de anticuerpos antigliadina y antiendomisio, con valores de inmunoglobulina sérica $A$ dentro de límites normales y le mencionaron que probablemente su bajo peso sea de origen genético.

\section{Pregunta que generó el caso}

En personas con delgadez ¿existen características clínicas o parámetros mensurables que ayuden a diferenciar a los pacientes sanos de aquellos que pudieran tener trastornos de la alimentación?

\section{Estrategia de búsqueda}

Se realizó una búsqueda en PubMed usando los siguientes términos: ('constitutional thinness' or 'underweigh' or 'thinness') AND ('eating disorder' or 'anorexia' or 'Feeding and Eating Disorders'[Mesh]). Se limitaron los resultados a revisiones sistemáticas y meta-análisis usando los filtros correspondientes. Se encontraron 25 citas, de las cuales se seleccionó una revisión sistemática que parecía responder a la pregunta planteada ${ }^{1}$.

\section{Algunos datos sobre la delgadez constitucional}

La delgadez constitucional es el estado de peso corporal disminuido -habitualmente definido como un IMC menor de 18,5- en el que el balance energético del metabolismo es neutro, es decir, los ingresos calóricos se equiparan con las pérdidas ${ }^{1}$.

Las personas con delgadez constitucional suelen presentar un peso habitual reducido pero estacionario en el tiempo, con dificultad para aumentarlo incluso luego de someterse a dietas hipercalóricas. A diferencia de otras entidades, este tipo de delgadez no suele acompañarse de trastornos de alimentación, enfermedades sistémicas, desnutrición o sobre-ejercitación. Sin embargo, su presencia suele despertar preocupación y motivar la búsqueda de causas patológicas asociadas, tanto por parte de los pacientes como de los profesionales de la salud ${ }^{2}$. Esto suele conducir a la estigmatización de la población afectada, siendo la anorexia nerviosa el principal diagnóstico diferencial con el cual se la suele confundir.

\section{Resumen de la evidencia}

Bailly $M$, et al. Is constitutional thinness really different from anorexia nervosa? A systematic review and meta-analysis. Rev Endocr Metab Disord. 2021 Apr 30. doi:

10.1007/s11154-021-09650-4. Online ahead of print.

El objetivo de esta revisión sistemática fue mejorar la caracterización diagnóstica de los pacientes con delgadez constitucional ${ }^{1}$.

\footnotetext{
a Servicio de Medicina Familiar y Comunitaria, Hospital Italiano de Buenos Aires. gabriel.villalon@hospitalitaliano.org.ar
} 


\section{Estrategia de búsqueda y selección de estudios}

Los autores realizaron una búsqueda en PubMed - MEDLINE, Embase, la biblioteca Cochrane, Google Scholar, y Clinical Trials entre 1950 y junio de 2020, utilizando como principales palabras clave los términos 'constitution/al/ly', 'thin/ness', 'lean/ness', 'weight gain resistance'.

Fueron incluidos estudios de cualquier diseño, en inglés o francés, que incorporaron varones o mujeres adultos con delgadez constitucional, identificados por criterios específicos y que brindaron información para su caracterización. Fueron excluidos los estudios que enrolaron pacientes con delgadez vinculable a otras causas (trastornos de la alimentación, desnutrición, otras patologías o sobre-ejercitación).

\section{Obtención y análisis de los datos}

Los autores mantuvieron un criterio amplio en el abanico de variables a extraer de los estudios primarios. Dentro de las categorías más específicas, incluyeron: los resultados principales relacionados con la antropometría/composición corporal, la ingesta y el gasto calórico, algunos parámetros bioquímicos y de regulación hormonal del apetito.

\section{Resultados principales}

Sobre un total de 1.212 artículos identificados, la revisión incluyó 39 estudios para el análisis cualitativo y 29 para la síntesis cuantitativa. Los diseños incluyeron estudios descriptivos de corte transversal, estudios analíticos de cohorte y ensayos clínicos no aleatorizados, con un total de 3.390 pacientes con delgadez constitucional que tenían una media de edad de 23,2 años. La Tabla 1 resume sus principales resultados.

\section{Fuente de financiamiento/conflicto de interés de los autores}

Los autores no recibieron financiamiento específico y declararon no tener conflictos de interés.

\section{Conclusiones de los autores}

Los pacientes con delgadez constitucional presentan un fenotipo atípico altamente diferenciable del de los pacientes con anorexia nerviosa.

\section{Conclusiones y recomendaciones}

Según la Organización Mundial de la Salud, el rango normal del IMC oscila entre 18,5 y $24,9 \mathrm{~kg} / \mathrm{m}^{2}$. Por debajo de este umbral se reconocen tres grados de delgadez: grado 1 o leve (IMC 17 a 18,49), grado 2 o moderada (IMC 16 a 16,9) y grado 3 o severa (IMC menor de 16$)^{3}$. A nivel global, según datos de 2016, alrededor de un $8,9 \%$ de la población adulta presentó un IMC menor de 18,5. Para Argentina, esta cifra ronda el $0,9 \%$ (0,4\% y $1,4 \%$ para varones y mujeres, respectivamente) ${ }^{4}$. Sin embargo, dentro de este grupo, la delgadez constitucional es una entidad poco estudiada, y resulta difícil conocer su prevalencia real ${ }^{5}$.

Una reciente revisión sistemática se propuso revisar los criterios utilizados para definir la presencia de delgadez constitucional $^{2}$. A partir de la bibliografía sintetizada, los autores proponen un algoritmo para la identificación clínica de esta condición, mediante la exclusión de pacientes que presenten alguna de las siguientes características: carencias nutricionales vinculadas con un bajo nivel socioeconómico; trastornos de la conducta alimentaria; enfermedades sistémicas; sobre-ejercitación; ausencia de ciclos menstruales; ausencia de intentos frustros para aumentar de peso; variaciones en el peso histórico.

Los trastornos de la conducta alimentaria representan como entidad el principal diagnóstico diferencial en los pacientes en quienes se advierte o consultan por un peso corporal disminuido. Entre ellos, la anorexia nerviosa suele representar el diagnóstico principal a tener en cuenta. Según el DSM en su última revisión (5ta. edición), si bien existen una gran variedad de síntomas y signos alternativos, para establecer el diagnostico de anorexia nerviosa se requiere la presencia de tres criterios principales: 1) restricción del ingreso calórico que conlleva un descenso de peso; 2) miedo intenso o desproporcionado a aumentar de peso; 3) alteración de la imagen corporal $^{6}$. Cuando éstos no se cumplen en su totalidad, pero existe aún sospecha de un trastorno de la alimentación, en la práctica suele emplearse el término de trastorno de la alimentación no especificado (o TANE) ${ }^{7}$.

Otros diagnósticos alternativos a considerar en el abanico de trastornos de la conducta alimentaria son el trastorno evitativorestrictivo de la ingesta, la ingesta compulsiva y la bulimia nerviosa; aunque estos dos últimos por lo general no cursan con delgadez 6,7

Si bien el diagnóstico de los trastornos alimentarios se define por criterios clínicos, resulta atractiva la posibilidad de contar con herramientas cuantitativas para descartar su presencia cuando la clínica resulta confusa. En este sentido, de la revisión sistemática de Bailly y col. ${ }^{1}$, se desprenden varias determinaciones que se comportarían de manera diferencial entre las personas con delgadez constitucional y aquellas que presentan anorexia nerviosa, como los dosajes de leptina, T3 libre, factor de crecimiento similar a la insulina 1(IGF-1), estradiol, las hormonas folículoestimulante y luteinizante en ayunas, y el cortisol de 24 horas. Sin embargo, es necesario tener en cuenta las limitaciones importantes del estudio que podrían restringir la extrapolación de sus resultados: la inconsistencia entre los estudios incluidos, el bajo tamaño muestral para muchas variables, y la muy baja tasa de varones incluidos en los estudios primarios (27 de los 39 estudios seleccionados incluyeron exclusivamente mujeres).

La utilidad de la leptina y otras citocinas para el diagnóstico diferencial de la delgadez constitucional y la anorexia nerviosa fue también indagada por los autores de otra revisión sistemática ${ }^{8}$, quienes concluyeron que, aunque se observen niveles medios de leptina más bajos en las personas con anorexia nerviosa cuando se las compara con las mujeres con delgadez constitucional, existe una superposición en los niveles individuales entre ambos grupos y, por lo tanto, no se pueden utilizar para diferenciar entre estos estados.

Por su parte, los autores de un estudio descriptivo que buscó diferenciar a las personas con delgadez constitucional de aquellas con anorexia nerviosa mediante el análisis y la comparación de una batería de marcadores bioquímicos similares a los estudiados en la revisión sistemática resumida ${ }^{1}$, concluyeron que, teniendo en cuenta la influencia del uso concomitante de anticonceptivos y las variaciones circadianas inherentes a varios marcadores, sumado a un mejor perfil de desempeño en las curvas de area bajo la curva (ROC), su mayor accesibilidad y su menor costo, la determinación de T3 libre podría resultar una determinación prometedora $^{9}$.

El caso presentado en la viñeta se interpretó hasta el momento como el de una paciente de delgadez constitucional. Preocupa un poco, sin embargo, el último descenso de peso, no habitual en su historial, pero que se encuentra relacionado con una intervencion quirúrgica reciente y en ausencia de otras alteraciones de riesgo. 
Tabla 1. Comparación de diferentes variables asociadas a antropometría/composición corporal, ingesta y gasto calórico, parámetros bioquímicos y regulación hormonal del apetito entre pacientes con delgadez constitucional, anorexia nerviosa y controles sanos. Notas: AN: anorexia nerviosa; DC: delgadez constitucional; DME: diferencias de media estandarizada; IC: intervalo de confianza; IMC: índice de masa corporal; DMO: densidad mineral osea; T3: triyodotironina; T4: tiroxina; IGF-1: factor de crecimiento similar a la insulina 1; FSH: hormona foliculo estimulante; LH: hormona luteinizante; ${ }^{* *}$ Denota las variables que además no presentaron superposición entre los valores extremos de los grupos con DC y AN. ND: valor no disponible.

\begin{tabular}{|c|c|c|c|c|c|}
\hline Variable & $\begin{array}{l}\text { Delgadez constitucio- } \\
\text { nal }\end{array}$ & Anorexia nerviosa & Controles & $\begin{array}{l}\text { DME (IC } 95 \%) \\
\text { entre DC y AN }\end{array}$ & $\begin{array}{l}\text { Diferencia entre } \\
\text { los grupos } \\
\end{array}$ \\
\hline Edad, años (IC $95 \%$ ) & $23,2(22,3$ a 24,0$)$ & $21,8(20,6$ a 23,1$)$ & $22,7(21,8$ a 23,7$)$ & $0,21(-0,00$ a 0,42$)$ & \\
\hline $\mathrm{IMC}, \mathrm{Kg} / \mathrm{m}^{2}(\mathrm{IC} 95 \%)$ & $16,7(16,4$ a 17,0$)$ & $15,1(14,7$ a 15,5$)$ & $21,6(21,2$ a 21,9$)$ & $1,12(0,75$ a 1,49$)$ & $\mathrm{AN}<\mathrm{DC}<\mathrm{C}^{* *}$ \\
\hline $\begin{array}{l}\% \text { grasa corporal (IC } \\
95 \% \text { ) }\end{array}$ & $19,5(18,0$ a 21,1$)$ & $11,7(9,7$ a 13,7$)$ & $27,1(25,5$ a 28,8$)$ & $1,59(1,18$ a 2,01$)$ & $\mathrm{AN}<\mathrm{DC}<\mathrm{C}^{* *}$ \\
\hline $\begin{array}{l}\text { Masa magra, kg (IC } \\
95 \%)\end{array}$ & $34,2(33,3$ a 35,1$)$ & $34,9(34,2$ a 35,6$)$ & $39,7(38,3$ a 41,0$)$ & $-0,24(-0,54$ a 0,06$)$ & $\mathrm{DC}=\mathrm{AN}<\mathrm{C}$ \\
\hline $\begin{array}{l}\text { Densidad mineral ósea, } \\
\mathrm{g} / \mathrm{cm}^{2}(I C 95 \%)\end{array}$ & $1,063(1,028$ a 1,099$)$ & $1,076(1,035$ a 1,117$)$ & $1,131(1,108$ a 1,153$)$ & ND & $\mathrm{DC}<\mathrm{C}$ \\
\hline $\begin{array}{l}\text { Ingreso energético total, } \\
\text { kcal/día (IC 95\%) }\end{array}$ & 1.931 (1.837 a 2.025) & $1.145(1.023$ a 1.267$)$ & $1.832(1.733$ a 1.932$)$ & $2,20(0,47$ a 3,93$)$ & $\mathrm{AN}<\mathrm{C}=\mathrm{DC} * *$ \\
\hline $\begin{array}{l}\text { Gasto energético total, } \\
\text { kcal/día (IC 95\%) }\end{array}$ & 1.782 (1.574 a 1.990$)$ & $1.912(1.501$ a 2.323$)$ & 2.018 (1.940 a 2.095) & ND & $D C<C$ \\
\hline $\begin{array}{l}\text { Gasto energético basal, } \\
\text { kcal/día (IC 95\%) }\end{array}$ & $1.104(1.062$ a 1.147$)$ & 908 (854 a 962) & $1.276(1.216$ a 1.336$)$ & $1,35(1,00$ a 1,71$)$ & $\mathrm{AN}<\mathrm{DC}<\mathrm{C}^{* *}$ \\
\hline $\begin{array}{l}\text { Gasto energético total } \\
\text { /Masa magra, kcal/- } \\
\text { día/kg (IC 95\%) }\end{array}$ & $33,35(30,09$ a 36,61$)$ & $27,19(25,27$ a 29,12$)$ & $31,62(29,07$ a 34,17$)$ & $1,33(0,79$ a 1,87$)$ & $\mathrm{AN}<\mathrm{C}<\mathrm{DC}$ \\
\hline $\begin{array}{l}\text { Leptina en ayunas, } \\
\mathrm{ng} / \mathrm{mL} \text { (IC } 95 \%)\end{array}$ & $5,8(4,9$ a 6,7$)$ & $2,0(1,6$ a 2,3$)$ & $10,6(9,2$ a 12,0$)$ & $1,51(1,21$ a 1,82$)$ & $\mathrm{AN}<\mathrm{DC}<\mathrm{C}^{* *}$ \\
\hline $\begin{array}{l}\text { Grelina en ayunas, } \\
\mathrm{pg} / \mathrm{mL}(\mathrm{IC} 95 \%)\end{array}$ & 472 (272 a 671) & 1.072 (621 a 1.522) & 761 (436 a 1086) & $-1,08(-1,62$ a $-0,53)$ & $\mathrm{DC}=\mathrm{C}<\mathrm{AN}$ \\
\hline $\begin{array}{l}\text { T3 libre en ayunas, pmo- } \\
\text { I/L (IC 95\%) }\end{array}$ & $4,16(3,99$ a 4,34$)$ & $2,88(2,69$ a 3,06$)$ & $4,12(3,78$ a 4,47$)$ & $2,90(2,09$ a 3,70$)$ & $\mathrm{AN}<\mathrm{C}<\mathrm{DC} * *$ \\
\hline $\begin{array}{l}\text { T4 libre en ayunas, pmo- } \\
\text { I/L (IC } 95 \%)\end{array}$ & $16,76(15,75$ a 17,77$)$ & $14,79(13,23$ a 16,34$)$ & $17,57(15,32$ a 19,82$)$ & $0,88(0,50$ a 1,26$)$ & $\mathrm{AN}<\mathrm{DC}=\mathrm{C}$ \\
\hline $\begin{array}{l}\text { Cortisol } 24 \mathrm{~h}, \mathrm{nmol} / \mathrm{L} \text { (IC } \\
95 \% \text { ) }\end{array}$ & $229,5(219,4$ a 239,5$)$ & $398,5(361,5$ a 435,4$)$ & $263,1(251,9$ a 274,3$)$ & $-2,56(-3,85 a-1,26)$ & $\mathrm{DC}<\mathrm{C}<\mathrm{AN}^{* *}$ \\
\hline $\begin{array}{l}\text { IGF-1 en ayunas, ug/L } \\
\text { (IC } 95 \%)\end{array}$ & $260,5(243,2$ a 277,9$)$ & $148,7(134,1$ a 163,4$)$ & $260,5(244,1$ a 276,9$)$ & $2,12(1,13$ a 3,10$)$ & $\mathrm{AN}<\mathrm{C}=\mathrm{DC} \mathrm{C}^{* *}$ \\
\hline $\begin{array}{l}\text { Estradiol en ayunas, } \\
\text { ng/L (IC 95\%) }\end{array}$ & $62,3(52,7$ a 72,0$)$ & $12,3(10,6$ a 14,0$)$ & $54,8(47,4$ a 62,3$)$ & $2,02(1,36$ a 2,69$)$ & $\mathrm{AN}<\mathrm{C}=\mathrm{DC}^{* *}$ \\
\hline $\begin{array}{l}\text { FSH en ayunas, IU/L (IC } \\
95 \%)\end{array}$ & $5,51(3,63$ a 7,40$)$ & $2,84(2,07$ a 3,60$)$ & $4,76(3,11$ a 6,42$)$ & $1,10(0,32$ a 1,88$)$ & $\mathrm{AN}<\mathrm{C}=\mathrm{DC}^{* *}$ \\
\hline $\begin{array}{l}\text { LH en ayunas, IU/L (IC } \\
95 \%)\end{array}$ & $10,08(8,15$ a 12,00$)$ & $1,31(0,65$ a 1,97$)$ & $8,56(6,83$ a 10,29$)$ & $2,04(1,04$ a 3,04$)$ & $\mathrm{AN}<\mathrm{C}=\mathrm{DC}^{* *}$ \\
\hline
\end{tabular}

Recibido el 17/11/2021 Aceptado el 19/12/2021 y Publicado el 22/12/2021.

\section{Agradecimientos}

A Silvina Dell'era por sus aportes como revisora de pares de este manuscrito.

\section{Referencias}

1. Bailly M, Boscaro A, Pereira B, et al. Is constitutional thinness really different from anorexia nervosa? A systematic review and meta-analysis. Rev Endocr Metab Disord. 2021; Available from: 10.1007/s11154-021-09650-4.

2. Bailly M, Germain N, Galusca B, et al. Definition and diagnosis of constitutional thinness: a systematic review. Br J Nutr. 2020;124(6):531-547. Available from: $10.1017 /$ S0007114520001440.

3. on Physical Status: the Use WEC, of Anthropometry I, editors. Physical status: the use and interpretation of anthropometry: report of a WHO Expert Committee. Geneva: World Health Organization; 1995.

4. World Health Organization. Prevalence of underweight among adults, BMI 18.5 (age-standardized estimate) (\%); $2021 . \quad$ Available from: https://www.who.int/data/gho/data/indicators/indicator-details/GHO/prevalence-of-underweight-among-adults-bmi-18-(age-standardizedestimate)-(-) [Last access: 2021-12-21].

5. Estour B, Galusca B, Germain N. Constitutional Thinness and Anorexia Nervosa: A Possible Misdiagnosis? Front Endocrinol (Lausanne). 2014;5:175 Available from: 10.3389/fendo.2014.00175 
6. D K, E A. UpTo Date. Anorexia nervosa in adults: Clinical features, course of illness, assessment, and diagnosis. Waltham, MA; 2021. Available from: https://www.uptodate.com/contents/anorexia-nervosa-in-adults-clinical-features-course-of-illness-assessment-and-diagnosis?search= anorexia\%20nerviosa\&source=search_result\&selectedTitle=6 118\&usage_type=default\&display_rank=6.

7. Yager J. UpTo Date. Eating disorders: Overview of epidemiology, clinical features, and diagnosis. Waltham, MA; $2021 . \quad$ Available from: https:/www.uptodate.com/contents/eating-disorders-overview-of-epidemiology-clinical-features-and-diagnosis?search=unspecified\% 20eating\%20disorder\&source=search_result\&selectedTitle=1 150\&usage_type=default\&display_rank=1\# 439450 .

8. Karageorgiou V, Furukawa TA, Tsigkaropoulou E, et al. Adipokines in anorexia nervosa: A systematic review and meta-analysis. Psychoneuroendocrinology. 2020;112:104485. Available from: 10.1016/j.psyneuen.2019.104485.

9. Estour B, Marouani N, Sigaud T, et al. Differentiating constitutional thinness from anorexia nervosa in DSM 5 era. Psychoneuroendocrinology. 2017;84:94-100. Available from: 10.1016/j.psyneuen.2017.06.015. 